\title{
Research on the Multi-channel P-persistent CSMA Protocol with Monitoring Function
}

\author{
Yingying Guo ${ }^{1,2}$, Jing $\mathrm{Nan}^{3}$, Hongwei Ding ${ }^{2}$, Yifan $\mathrm{Zhao}^{2}$ and Shengjie Zhou ${ }^{2}$ \\ ${ }^{1}$ Yunnan Radio Monitoring Station of Stat e Radio Monitoring Center, Kunming \\ ${ }^{2}$ School of Informaton, Yunnan University, Kunming, China \\ ${ }^{3}$ Xianyang, Branch of China Mobile Communication Co.Ltd, Shanxi, China \\ *dhw1964@163.com
}

\begin{abstract}
This paper presents a new sensor network MAC protocol: the multi-channel $p$ persistent CSMA protocol with monitoring function (ACKPPCMA) from the perspective of improving the channel utilization and meeting the demand of the high QoS for high priority. Analyze the model by using the average cycle method. The Computer simulation results verify the correctness of the theory. Has some practical significance.
\end{abstract}

Keywords: Multi-Channel, p -Persistent CSMA, Monitoring Function, Throughput, Priority

\section{Introduction}

Wireless sensor network (WSN) [1, 2] has a strong correlation of applications. That is, for different applications, need to adopt different strategies, in order to achieve the purpose that the developed wireless sensor network can fit the applications well. With the progress of the times, the applications of wireless sensor network in every aspect will be increased, and some of the existing protocol cannot meet these requirements well, so need to introduce some of the new protocols.

From the angle of increasing wireless communication service quality [3] and make the different service having different service quality requirement, this paper analyzes the multi-channel $p$-persistent CSMA protocol with monitoring function (ACKPPCMA) using the average cycle analysis method [4,5]. Adding a monitoring signal ACK to reduce the collision probability and improve the channel utilization and communication security [6]; through the introduction of multi-channel mechanism [7] to meet the demand of the high QoS [8] for high priority, but also to ensure the fairness of the system.

\section{The Analysis of ACKPPCMA Protocol}

\subsection{The Description of Protocol Model}

Consider the system having $\mathrm{N}$ channels and $\mathrm{N}$ priorities, the nodes are accessed to the channels randomly by the business priorities of themselves [9]. Assume that the priority sequence is arranged from low to high as priority 1 , priority $2 \ldots$ priority $\mathrm{N}$ [10]. The service with priority $i$ occupies channel 1 to $i(i=1,2 \ldots \mathrm{N})$, that is the service with priority 1 occupies channel 1 , priority 2 occupies channel 1 and channel 2 , and the service with priority $\mathrm{N}$ occupies channel 1 to channel $\mathrm{N}$.as shown in Figure 1. The arrival information packets on the channel $i$ subject to the Poisson distribution [11] with arriving rate $G_{i}$, the arrival packets of priority $r$ on the channel $i$ subject to the 
Poisson distribution with arriving rate $\lambda_{i}=G_{i} /(N-i+1)$. At this point, the system load balancing, the arrival rate of each channel rate is $G_{i}=G(i=1,2, \ldots, N)$.

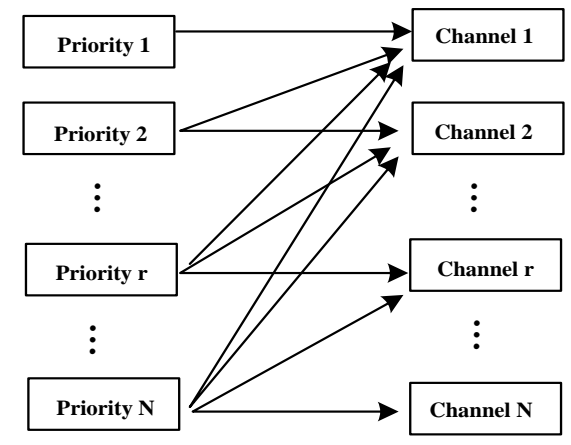

\section{Figure 1. The System Model of ACKPPCMA Protocol}

Each user detect channel state before they send the information packets in the channel, if the channel is idle, user persist in sending packets by probability of $p$, and give up by probability of $(1-p)$.Since it has transmission delay in channels, perhaps more than one user will detect that it is in the idle channel state at the same time, and persist in sending by probability of $p$ at the next timeslot, then, those information packets in this channel will collide with each other, they will be sent again after stepping back several timeslots randomly.

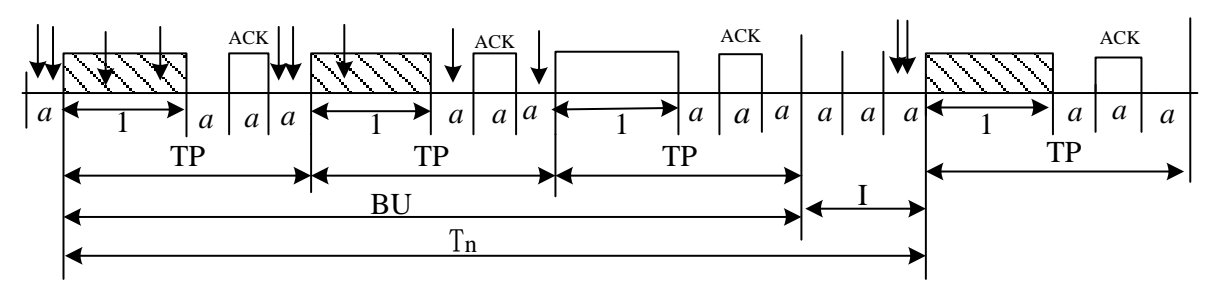

Figure 2. The Channel Model of ACKPPCMA Protocol

\subsection{The Analysis of System Throughput}

We adopt the method of average cycle analysis that put forward by Professor Dongfeng Zhao when we analyze the model mentioned above:

Event that information packets sent successfully $(U)$;

Event that information packets collide with each other $(B)$;

Event that there are no information packets in the channel arrive, the channel is idle ( $I$ ) ;

According to the Figure 2, we can divide these successful event $U$, collision event $B$ and idle event $I$ into two categories essentially, in order to analyze easily we also divide the state of channel into two kinds, one kind called idle period is the time occupied by successive several idle event $I$, the other called busy circle period is the time occupied by successful event $U$ and collision event $B$, while these two states will appear constantly.

In order to analyze easily, before making an analysis of system performance, we make assumptions about wireless communication system below: 
(1) Assuming that channels are in the ideal state, there is no noise interference in channels;

(2) Assuming that the maximum transmission delay time is $a$, namely, the length of timeslot of the channel is $a$, the length of information packet is unit length 1 , and it is integral multiple of the length of timeslot $a$;

(3) The timeline of system is divided according to unit length of timeslot, the packets arrived at any timeslot will be sent at the starting time of next timeslot;

(4) The access method of number $i(i=1,2, \ldots, N)$ channel is timeslot formula ppersistent CSMA protocol, and the arrival process of number $i$ channel satisfy the Poisson process whose independent parameter is $G_{i}$, each arrival process on the channel is independent of each other;

(5) The information packet need to send at the first timeslot (time delay $a$ ) in transmission period $T P$ can always detect the state of channel successfully;

(6) The collision information packets will be sent again after stepping back several timeslots randomly, and they have no influence on their arrival process.

The probability of no packet deciding to be sent in idle timeslot $a$ in channel $i$ is

$$
q_{a}^{0}=e^{-G_{i} p a}
$$

The probability of only one packet deciding to be sent at idle timeslot $a$ in channel $i$ is

$$
q_{a}^{1}=G_{i} p a e^{-G_{i} p a}
$$

The probability of no packet deciding to be sent in $(1+3 a)$ is

$$
q_{1+3 a}^{0}=e^{-G_{i} p(1+3 a)}
$$

The probability of only one information packet deciding to be sent in $(1+3 a)$ is

$$
q_{1+3 a}^{1}=G_{i} p(1+3 a) e^{-G_{i} p(1+3 a)}
$$

The probability of $h$ events are continuously idle in channel $i$ is

$$
p\left(N_{I}=h\right)=\left(e^{-G_{i} p a}\right)^{h-1}\left(1-e^{-G_{i} p a}\right)
$$

The probability of $k$ events are continuously busy in channel $i$ is

$$
p\left(N_{B U}=k\right)=e^{-G_{i} p(1+3 a)}\left(1-e^{-G_{i} p(1+3 a)}\right)^{k-1}
$$

So the joint probability [12] of $h$ events are continuously idle and $k$ events are busy in channel $i$ in a cycle period is

$$
p\left(N_{I}=h, N_{B U}=k\right)=\left(e^{-G_{i} p a}\right)^{h-1}\left(1-e^{-G_{i} p a}\right) e^{-G_{i} p(1+3 a)}\left(1-e^{-G_{i} p(1+3 a)}\right)^{k-1}
$$

According to the expected value of joint probability distribution above, we can calculate that the average number of timeslots occupied by channel $i$ which is idle in a cycle period is

$$
E\left(N_{I}\right)=\sum_{h=1}^{\infty} \sum_{k=1}^{\infty} h p\left(N_{I}=h, N_{B U}=k\right)=\frac{1}{1-e^{-G_{i} p a}}
$$

The average number of timeslots occupied by channel $i$ which is busy in a cycle period is 


$$
E\left(N_{B U}\right)=\sum_{h=1}^{\infty} \sum_{k=1}^{\infty} k p\left(N_{I}=h, N_{B U}=k\right)=\frac{1}{e^{-G_{i} p(1+3 a)}}
$$

Before calculating the average number of timeslots occupied by information packet sending successful event $U$ in a cycle period $T_{i}$, we first give a definition below:

$U_{1}$ : There are information packets arriving at the last timeslot in idle period, and among them, there is only one packet deciding to be sent by probability of $p$, so this packet will be transmitted successfully at the next timeslot.

$U_{2}$ : There are information packets arriving in busy period, and all these arrived packets will detect the state of channel $i$ continuously until channel $i$ becomes idle, but there is only one packet deciding to be sent by probability of $p$ when channel $i$ is idle, this packet will be transmitted successfully in the next transmission cycle period $T P$.

The average number of timeslots occupied by time $U_{1}$ in one cycle period $T_{i}$ is:

$$
E\left(N_{U_{1}}\right)=\frac{G_{i} p a e^{-G_{i} p a}}{1-e^{-G_{i} p a}}
$$

The average number of timeslots occupied by time $U_{2}$ in one cycle period $T_{i}$ is:

$$
E\left(N_{U_{2}}\right)=G_{i} p(1+3 a)
$$

So the average number of timeslots occupied by event $U$ that information packets sent successfully in channel $i$ in one cycle period $T_{i}$ is:

$$
E\left(N_{U}\right)=\frac{G_{i} p a e^{-G_{i} p a}}{1-e^{-G_{i} p a}}+G_{i} p(1+3 a)
$$

According to the analysis, the available length of timeslots occupied by event $U$ that information packets sent successfully in channel $i$ in one cycle period $T_{i}$ is:

$$
E\left(U_{i}\right)=\frac{G_{i} p a e^{-G_{i} p a}}{1-e^{-G_{i} p a}}+G_{i} p(1+3 a)
$$

The available timeslots length of $B U$ in channel $i$ in one cycle period $T_{i}$ is:

$$
E\left(B U_{i}\right)=\frac{1+3 a}{e^{-G_{i} p(1+3 a)}}
$$

The available timeslots length of idle event in channel $i$ in one cycle period $T_{i}$ is:

$$
E\left(I_{i}\right)=\frac{a}{1-e^{-G_{i} p a}}
$$

According to the computational formula of the systemic throughput:

$$
S_{i}=\frac{E\left(U_{i}\right)}{E\left(B U_{i}\right)+E\left(I_{i}\right)}
$$

We can get the throughput of p-persistent CSMA control protocol with monitoring function in the number $i$ channel: 


$$
S_{i}=\frac{e^{-G_{i} p(1+3 a)}\left[G_{i} p a e^{-G_{i} p a}+G_{i} p(1+3 a)\left(1-e^{-G_{i} p a}\right)\right]}{(1+3 a)\left(1-e^{-G_{i} p a}\right)+a e^{-G_{i} p(1+3 a)}}
$$

In the $N$ channels of wireless communication system, because this channel model is a load equilibrium model, so the arrival probabilities of each channel are the same, that is to say:

$$
G_{1}=G_{2}=G_{3}=\cdots=G_{i}=\cdots=G_{N}=G
$$
[13]:

Basing on the above analysis and computational formula of the systemic throughput

$$
S=\sum_{i=1}^{N} \frac{E\left(U_{i}\right)}{E\left(B U_{i}\right)+E\left(I_{i}\right)}
$$

The systemic throughput of multi-channel $p$-persistent CSMA protocol with monitoring is:

$$
S=N S_{i}=\frac{N e^{-G p(1+3 a)}\left[G p a e^{-G p a}+G p(1+3 a)\left(1-e^{-G p a}\right)\right]}{(1+3 a)\left(1-e^{-G p a}\right)+a e^{-G p(1+3 a)}}
$$

The arrival probability of the business with the priority $l$ in channel $i$ is:

$$
\lambda_{i}=\frac{G_{i}}{N-i+1}(r \leq l)
$$

Assuming that the length of information packet sent by the business with priority $l$ successfully in the average cycle period of channel $i$ is $E\left(U_{i}^{(p l)}\right)(r \leq l)$.

Then according to the above analysis, we can get the throughput of the p-persistent CSMA protocol with monitoring function with the priority $l$ :

$$
S_{p l}=\left(\sum_{i=1}^{l} \frac{1}{N-i+1}\right) \frac{e^{-G p(1+3 a)}\left[G p a e^{-G p a}+G p(1+3 a)\left(1-e^{-G p a}\right)\right]}{(1+3 a)\left(1-e^{-G p a}\right)+a e^{-G p(1+3 a)}}
$$

\subsection{The Analysis of System Throughput}

On the premise of ACK acknowledge signal can always be transmitted correctly, the retransmitted average time delay time $\mathrm{R}$ include information packet transmission time 1 , ACK signal transmission time $a$, back and forth transmitted time delay $2 a$, average retransmission time delay $\delta$, so $R=1+3 a+\delta$. And because $(G / S-1)$ is the average value of the number of information packets need to retransmit, so the average time delay of information packet is :

$$
D=\left\{\frac{G(1+3 a)\left(1-e^{-G p a}\right)+a G e^{-G p(1+3 a)}}{N e^{-G p(1+3 a)}\left[G p a e^{-G p a}+G p(1+3 a)\left(1-e^{-G p a}\right)\right]}-1\right\} R+1+a
$$




\subsection{The Analysis of Protocol Energy Consumption}

There are three parts consist of the main energetic expense $[14,15]$ of the system in communication aspect: the energy consumed by detecting channel SPD, energy consumed by sending packets SPS and energy consumed by receiving packets SPR.

Assuming the transmitting power of sensor node is $P_{t x}$, receiving power is $P_{r x}$, the power of detection channel is $P_{d d}$.

For channel $i$,in a channel cycle period $T_{i}$, the number of busy cycle period $T P$ is $E\left(B U_{i}\right) /(1+3 a)$, while in each $T P$ of busy cycle period ,the number of terminal nodes of the channel need to be sent and persistently detected by information packets between time " 1 " and " $a$ " is $(1+a) G_{i}$, average detecting time is $1+2 a$; the number of terminal nodes of the channel need to be sent and persistently detected by information packets in time " $a$ " is $2 a p G$, average detecting time is $a$. So in $T_{i}$, the sum of energy consumed by all terminal nodes detecting channels of channel $i$ is

$$
S P D_{i}=\frac{G_{i}\left[(1+2 a)(1+a)+2 a^{2} p\right] P_{d d}}{e^{-[(1+a)+2 a p p] G_{i}}}
$$

At the same time, in the $T_{i}$, there are an average number of $E\left(U_{i}\right) /(1+a) T P$ having packets transmitted successfully, so the sum of energy consumed by information packets transmitted successfully in receiving channel $i$ is

$$
S P R_{i}=\left(\frac{G_{i} p a e^{-G_{i} p a}}{\left(1-e^{-G_{i} p a}\right)(1+3 a)}+G_{i} p\right) P_{\mathrm{r} x}
$$

In the first $T P$ of busy cycle period, the average number of packets sent in channel $i$ is:

$$
A S F_{i}=a G_{i} p
$$

And following $T P$, the average number of packets sent in each $T P$ in channel $i$ is:

$$
A S L_{i}=G_{i}(1+a+2 a p)
$$

So the sum of energy consumed by all terminal nodes sending information packets in channel $i$ is:

$$
S P S_{i}=\left[a p G_{i}+G_{i}(1+a+2 a p)\left(\frac{G_{i} p a e^{-G_{i} p a}}{\left(1-e^{-G_{i} p a}\right)(1+3 a)}+G_{i} p-1\right)\right] P_{t x}
$$

Because each channel model is a load equilibrium model, so in one channel cycle period, the sum of energy consumed by all nodes in system per unit time is:

$$
\begin{aligned}
E C A & =\sum_{i=1}^{N} \frac{S P D_{i}+S P R_{i}+S P S_{i}}{E\left(T_{i}\right.} \\
& =N\left\{\frac{G_{i}\left[(1+2 a)(1+a)+2 a^{2} p\right] P_{d d}}{e^{-[(1+a)+2 a p] G_{i}}}+\left(\frac{G_{i} p a e^{-G_{i} p a}}{\left(1-e^{-G_{i} p a}\right)(1+3 a)}+G_{i} p\right) P_{\mathrm{r} x}\right. \\
& \left.+\left[a p G_{i}+G_{i}(1+a+2 a p)\left(\frac{G_{i} p a e^{-G_{i} p a}}{\left(1-e^{-G_{i} p a}\right)(1+3 a)}+G_{i} p-1\right)\right] P_{t x}\right\} / E\left(T_{i}\right)
\end{aligned}
$$

Among them $E\left(T_{i}\right)=E\left(I_{i}\right)+E\left(B U_{i}\right)=\frac{1+3 a}{e^{-G_{i} p(1+3 a)}}+\frac{a}{1-e^{-G_{i} p a}}$. 


\section{Experiment Analysis}

On the basis of conclusion above, we make a computer simulation experiment on multi-channel $p$-persistent CSMA protocol with monitoring function, the experiment conditions are: the channel is in a ideal state of no noise, no disturbance; the arrival probability of channel is $G$,delay time is $a$, the length of information packet is 1 .

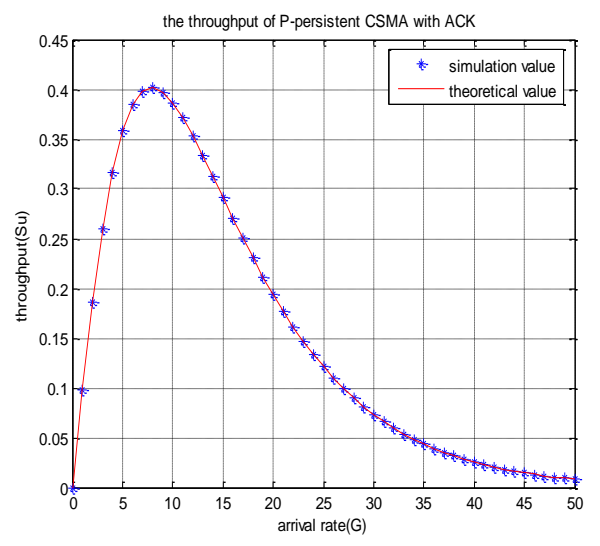

Figure 3. The Theoretical Values and Simulation Values of the ACKPPCMA Protocol

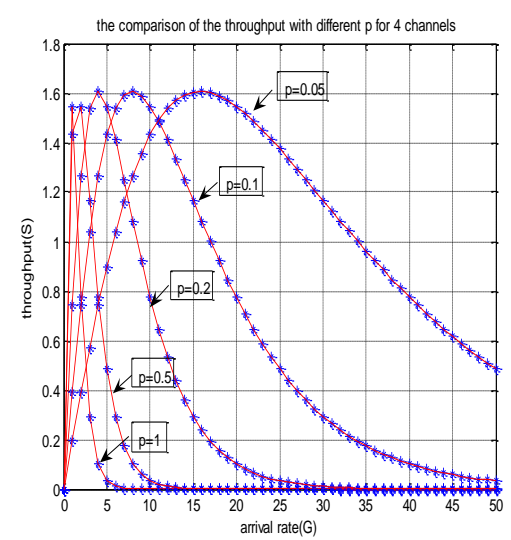

Figure 4. The Comparison of the System Throughput with Different $p$ for 4 Channels

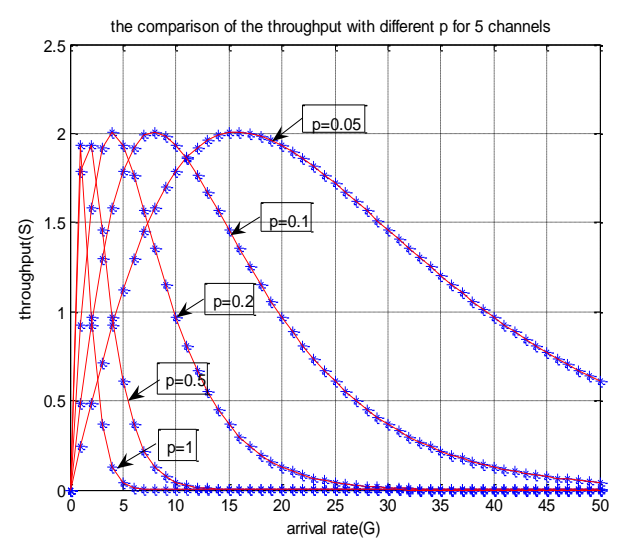

Figure 5. The Comparison of the System Throughput with Different $p$ for $\mathbf{5}$ Channels

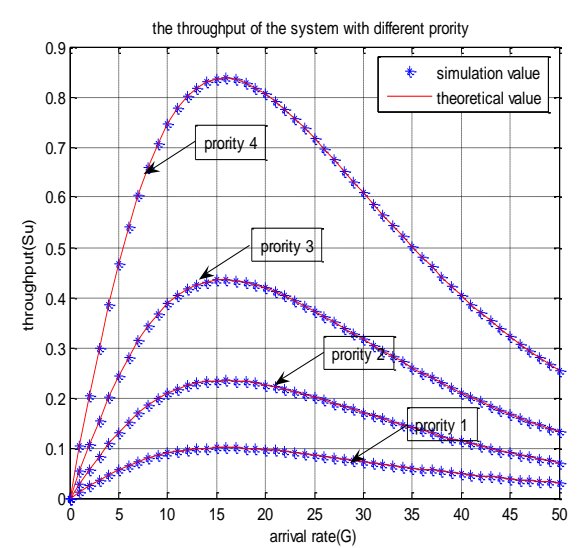

Figure 6. The Comparison of the Throughput of Each Priority for 4 Channels with $p=0.05$ 


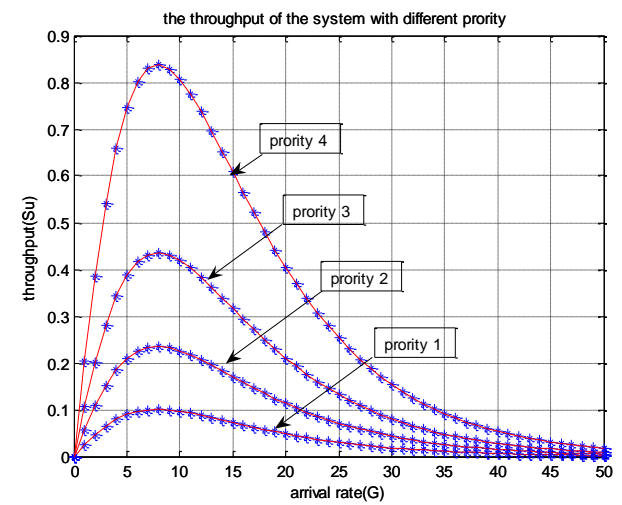

Figure 7. The Comparison of the Throughput of Each Priority for 4 Channels with $p=0.1$

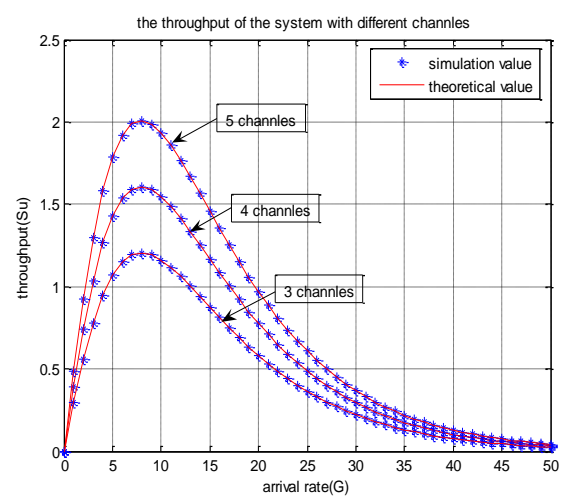

Figure 9. The Comparison of the System Throughput with Different Channels for $p=0.1$

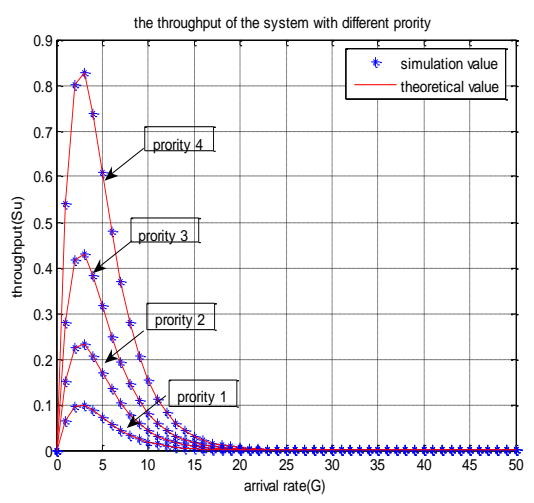

Figure 8. The Comparison of the Throughput of Each Priority for 4 Channels with $p=0.3$

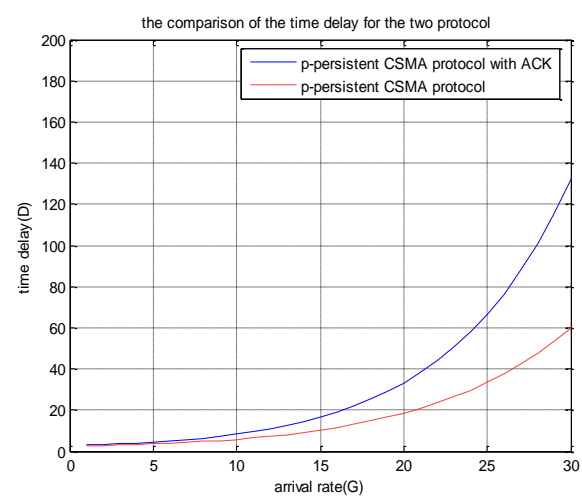

Figure 10. The Comparison of the System Delay for the Different Protocol For $a=0.1, p=0.1$

As can been seen from Figure 3, the theoretical values are well agree with the simulation values so that proved the correctness of the theoretical analysis. And under the control of the protocol, the system has a higher throughput in the light load, avoiding the waste of channel resources. But when the system load increases, the system throughput will decrease with the creasing of the collision probability.

As can be seen from Figure $4 \sim$ Figure 8, regardless of the amount of channels in system, systematic throughput and each priority throughput both will change when the system adopt different sending probability $p$. As can be seen from the figure, no matter it is systematic throughput or each priority throughput, viewed from trend of throughput of the curve, when the sending probability is large, the curve's ascent trend and descent trend both are relatively steep. When the sending probability is small, the curve's ascent trend and descent trend both are relatively slow, that is to say, the addition of sending probability (value $p$ ) will lead to overall falling of systematic throughput and priority throughput, this is because the larger the sending probability (value $p$ ) is, the more information packets will be decided to be sent when the channel is idle, this will result in the addition of the number of collision information packets, lead throughput to decrease. But, when systematic arrival probability get a less value, namely, systematic load is relatively light, the situation of systematic throughput with smaller sending probability is more than it with larger sending probability on the contrary, this is because in the state of systematic load is light, there are less information packets arrive in the system, so there are less information packets will be decided to be sent when the channel is idle, in this 
case the possibility of information packets collide with each other in channels decrease, so the probability of information packets sent successfully increase, but if it is a lower sending probability this time, originally there are few information packets arrive at in channels, while these packets giving up being sent with a probability of $(1-p)$, and $(1-p)$ is a very large probability, so this will lead channel to be idle and there is no information packets to be decided to be sent, thus causes a waste of resource of channels, reduces the systematic throughput. When the sending probability ( $p$ value ) gets a value of 1, that's to say the arrival packets once detect that channel is in the idle state they will immediately be sent, this time this strategy adopted can be considered to be multichannel 1-persistent CSMA with monitoring function. According to the analysis above we know that, in order to ensure the systematic throughput, it's very important to choose sending probability's value $p$, when businesses with same priority let probability $p$ take different values, the changes of throughput are also different, with the decreasing of probability $p$,the maximum value of throughput of businesses with same priority keeps unchanged, the average value will increase. So probability $p$ can influence channel throughput, if wants channels to get higher utilization rate, then it's very important to choose a suitable value $p$. As can been seen from the experiment result, when the load value $\mathrm{G}$ is a bigger value, we should choose the smaller value $p$, when the load value $\mathrm{G}$ is a smaller value, we should choose the bigger value $p$, this way can avoid network congestion, at the same time, it can also reduce the collision that caused by the competition among users and raise the throughput and channel utilization rate efficiently.

When the sending probability $p$ keeps unchanged, the throughput with different priority businesses in system has very obvious differences; the quota of the business throughput with low priority is less than it with high priority. When probability value $p$ is a constant value, in the system which has multi-channel $p$-persistent CSMA wireless local area networks MAC protocol with ACK, the business throughput with larger priority is always higher than it with smaller priority. But the business with low priority can also achieve channel resource, indicating that the system not only can satisfy the higher QoS requirement of business with high priority, but also doesn't invalidate the business with low priority, meeting each need of varied priority businesses. Figure 9 just explains this point, systematic throughput will also response to increase.

Illustrated by Figure 10, because there are ACK monitoring signals joining in multichannel $p$-persistent CSMA wireless local area networks MAC protocol with ACK, this causes system time delay to increase to some extent. But it decreases the probability of occurring systematic collision, increases the throughput, especially when the system is in a state of light load, systematic throughput performance index shows excellent.

\section{Conclusions}

This article analyzed a wireless sensor network MAC protocol based on random multiple access: the multi-channel $p$-persistent CSMA protocol with monitoring function (ACKPPCMA). By adding a monitoring signal ACK behind the system delay to increase the controllability of the system, reduce the collision probability and improve the channel utilization. Using the average cycle method to make a systematic modeling and mathematical modeling analysis on the ACKPPCMA protocol, gets the mathematical expressions of the system throughput, each priority traffic throughput, the average delay of information packets, the system energy consumption and other performance indicators through rigorous mathematical derivation. The Computer simulation results verify the correctness of the theory. At last, do computer simulation experiments according to work theory of the protocol, the computer simulation results verify the correctness of the theories. 


\section{Acknowledgments}

This work was supported by the National Natural Science Foundation of China (61072079); Natural Science Foundation of Yunnan Province (2010CD023); Graduate Scientific Research Fund of Yunnan University (ynuy201047).

\section{References}

[1] K. Pister, B. Hohlt, J. Jeong, L. Doherty and J. P. Vainio, "Ivy A Sensor Network Infrastructure [EB/OL]", http://www-base.eecs.berkeley.edu/projects/ivy, (2003).

[2] X. Zhang, "The Study on Wireless Sensor Network", Jiangnan University, (2009) February.

[3] L. Binbin, "The Analysis of Multi-channel Random Multiple Access Wireless Communication Network Protocol based on Probability Detection", Kunming: Yunnan University, (2006).

[4] D. Zhao, "Study on A New Method for Continuous-time Systems of Random Access Channel", Journal of Electronics, vol. 21, no. 1, (1999), pp. 37-41.

[5] D. Zhao, L. Bihai and Z. Sumin, "Study on a New Method for the Slotted Access Channel", Journal of Electronics, vol. 19, no. 6, (1997), pp. 814-819.

[6] B. S. Tsybakov and V. A. Mikhailov, "Ergodicity of a Slotted ALOHA System", Problemy Peredachi Informatsii, vol. 15 , no. 4, (1979), pp. 73-87.

[7] L. Pingyuan, H. Guoshen and L. Zuxin, "Communication Model of Random Multiple Address System With Multiple Channels", Journal of Jishou University (Natural Science Edition), vol. 24, no. 2, (2003), pp. 79-82.

[8] B. Cheng, L. Ci and C. Tian, "A Multi-channel MAC Protocol with High Throughput for Wireless Sensor Networks", Advanced Technologies in Ad Hoc and Sensor Networks, Springer Berlin Heidelberg, (2014), pp. 145-154.

[9] F. M. Di, A. J. Ghandour and H. Artail, "On the Impact of Multi-channel Technology on SafetyMessage Delivery in IEEE 802.11 p/1609.4 Vehicular Networks", Computer Communications and Networks (ICCCN), 2012 21st International Conference on. IEEE, (2012), pp. 1-8.

[10] J. He, "Research on the Access Protocol of Ad Hoc Network Based on the Multi-Channel", Modern Computer: half Edition, vol. 10, (2008), pp. 113-115.

[11] J. K. Min and Y. M. Kwon, "Priority Based Multi-Channel MAC Protocol for Real-Time Monitoring of Weapon Flight Test Using WSNs", 센서학회지, vol. 22, no. 1, (2013), pp. 18-27.

[12] E. Gulko, "Tree-Based Multi-Access Protocols Where Collision Multiplicities Are Known", IEEE Trans Commun, vol. 33, (1985), pp. 999.

[13] Z. Dongfeng, "Study on the Average Cycle Method for Slotted Multiple-Access Communications", Journal Of China Institute Of Communications, vol. 20, no. 8, (1999), pp. 80-85.

[14] A. Hoildi, J. D. Decotignie and J. Hernandez, "Low Power MAC Protocols for Infrastructure Wireless Sensor Networks", Proc. European Wireless (EW'04), Barcelona, Spain, vol. 2, (2004), pp. 563-569.

[15] A. El-Hoiydi and J. D. Decotignie, "WiseMAC: An Ultra Low Power MAC Protocol for the Downlink of structure Wireless Sensor Networks", the 9thInt'1 Symposium, On Computers and Communications Alexandria, Egypt, (2004).

\section{Authors}

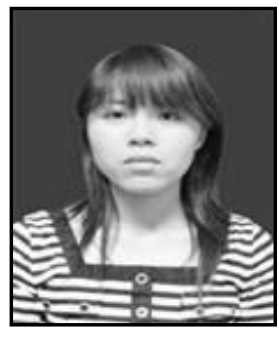

Yingying Guo, Female. Graduate from Yunnan University, master degree. Work in the Yunnan Radio Monitoring Station of the State Radio Monitoring Center. Mainly engaged in the research of computer network communications.

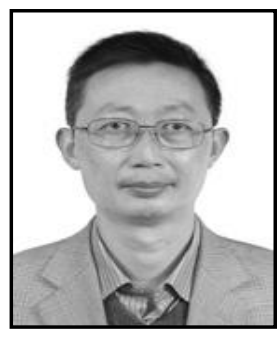

Hongwei Ding, Corresponding author. Male. Professor of Yunnan University, PhD Degree. Mainly engaged in the research of random multiple access communication system, polling system, network communication engineering. 